\title{
Cross-category phonological effects on ERP amplitude demonstrate context-specific processing during reading aloud
}

\author{
Jessie S. Nixon ${ }^{1,2,3}$, Jacolien van Rij ${ }^{4}$, Xiaoqing $\mathrm{Li}^{5}$, Yiya Chen ${ }^{1,2}$ \\ ${ }^{1}$ Leiden Institute for Brain and Cognition (LIBC), Leiden University, Netherlands \\ ${ }^{2}$ Leiden University Centre for Linguistics, Leiden University, Netherlands \\ ${ }^{3}$ MARCS Institute, University of Western Sydney, Australia \\ ${ }^{4}$ Institute of Psychology, Chinese Academy of Science, China \\ ${ }^{5}$ Department of Linguistics, University of Tübingen, Germany \\ https://doi.org/10.36505/ExLing-2015/06/0014/000251
}

\begin{abstract}
The phonetic realisation of speech sounds depends on their context: e.g., /t/ is aspirated in 'top', but unaspirated in 'stop'. Similarly, Beijing Mandarin Tone 3 (T3) usually has a low contour, but preceding another T3 syllablehas a rising contour (sandhiT3). Importantly, Tone 2 (T2) also has a similar rising contour. Yet how such phonetic variation is processed online during reading and speech production is not well understood. ERP amplitude was measured as native Mandarin speakers read aloud words preceded by a $48 \mathrm{~ms}$ masked prime. Prime and target always differed in tone category. Critical targets were T2-initial words. Primes were either sandhi T3 (contourmatch) or low T3 (mismatch). Generalised additive mixed models (GAMs) revealed a complex interaction between prime type, prime frequency and target frequency emerging around $100 \mathrm{~ms}$ and $300-400 \mathrm{~ms}$ following target presentation.In the contourmatch condition, when prime and target frequency were both high or both low, there was increased negativity, suggesting competition between prime and target.In the mismatch condition, there was relatively little effect of item frequencies. This difference in the pattern of effects between the contour-match and mismatch primes provides evidence for top-down effects of context on phonological processing of masked primes during reading aloud.
\end{abstract}

Key words: reading aloud, context effects. EEG / ERPs, Mandarin Chinese, masked priming, tone processing, allophonic variation.

\section{Introduction}

Phonetic variation is a fundamental property of speech. Speech sounds vary depending on their context. For example, in words like 'spin', where the first sound is $/ \mathrm{s} /$, the second sound is considered to belong to the same sound category $/ \mathrm{p} /$ as in the word 'pin'. But acoustically, the voice onset time falls between the /p/ of 'pin' and /b/ of 'bin'. How this kind of 'within-category' variation is processed is not yet well understood.

In Beijing Mandarin, Tone 3 (T3) has at least two variants. T3 usually has a low contour, but preceding another T3 syllable, it is realised with a rising contour (sandhi T3).Importantly for the present study, the contour of sandhi

ExLing 2015: Proceedings of 6th Tutorial and Research Workshop on Experimental Linguistics, 26-27 June 2015, Athens, Greece 
T3 is very similar to another tone, Tone 2.A previous study (Nixon, Chen \& Schiller, 2015) showed that production and visual processing of Mandarin tonal variants involves multi-level phonological processing, where there is activation of both the tonal contour and the tone category. The present study investigates whether the context provided by the following character in briefly presented masked primes affects processing of tone during reading aloud. In addition, it investigates whether phonological similarity (contour overlap) between prime and target can affectprocessing even when prime and target differ in tone category.

\section{Method}

\section{Participants}

Twenty-four native speakers of Beijing Mandarin participated in the experiment for pay.

\section{Design and stimuli}

Critical targets consisted of 25 two-character Chinese words, of which the initial character was Tone 2 (e.g. 鱼缸, yu2gang1, 'fish tank'). Each target was preceded by a two-character prime. The initial syllable of each prime was a Tone 3 character, which had the same segmental syllable as the first character of the target. Therefore, all prime-target pairs differed in terms of the tone category. The second character was either T3 (i.e. sandhi word, contourmatchprime, e.g. 雨水, yu3shui3, 'rain') or a different tone (i.e. low-tone word, mismatch prime, e.g. 雨衣, yu3yi1 'raincoat'). For each target word, the initial character of the prime was identical between prime conditions. They either matched or mismatched in contour.

\section{Procedure}

Stimulus presentation and reaction time data acquisition were conducted using the E-Prime 2.0 with a voice key trigger. The session began with a practice block to familiarise participants with the procedure. Each experimental trial began with a fixation cross with jittered presentation time (400-700 ms) to reduce time-induced expectancy waves. A forward-mask followed for100 ms, before presentation of the prime for 48 ms.A backward mask was presented for $17 \mathrm{~ms}$ to avoid images of the prime remaining on the retina. Finally, the target word was presented for a maximum of $2000 \mathrm{~ms}$ or until the participant response, which triggered the voice key and caused the word to disappear. The experimenter coded incorrect responses and voice key errors in a $1400 \mathrm{~ms}$ interval before the beginning of the next trial. Response time was calculated from the time of target word presentation until the voice key was triggered by the participant response. 


\section{Analysis and Results}

ERPs were analysed using Generalised Additive Mixed Modelling (Wood, 2006), a non-linear model with random effects for subjects and items. Analysis was conducted on a set of 24 electrodes evenly distributed over the scalp. To avoid potential speech artefacts, only data preceding $500 \mathrm{~ms}$ after target presentation were analysed. For 19 of the 24 electrodes, model comparisons and model summaries showed a significant interaction between prime type, prime frequency and target frequency over time. Figure 1 shows the interaction between prime frequency and target frequency over time for the mismatch (toprow) and contour-match conditions (bottom row) at electrode Fz.Each panel shows the interaction between Johnson transformed prime frequency and target frequency. ERP amplitude is colour-coded.

ERP amplitude varies between prime types (contour-match vs. mismatch) and interacts with prime and target word frequency. Divergences emerge around $100 \mathrm{~ms}$ and $300-400 \mathrm{~ms}$ after target presentation. In the contour-match condition, negativity is reduced when target frequency is relatively high and prime frequency low. However, when prime and target frequency are both high or both low, there is increased negativity, suggesting competition between prime and target. In the mismatch condition, there is relatively little effect of item frequencies.

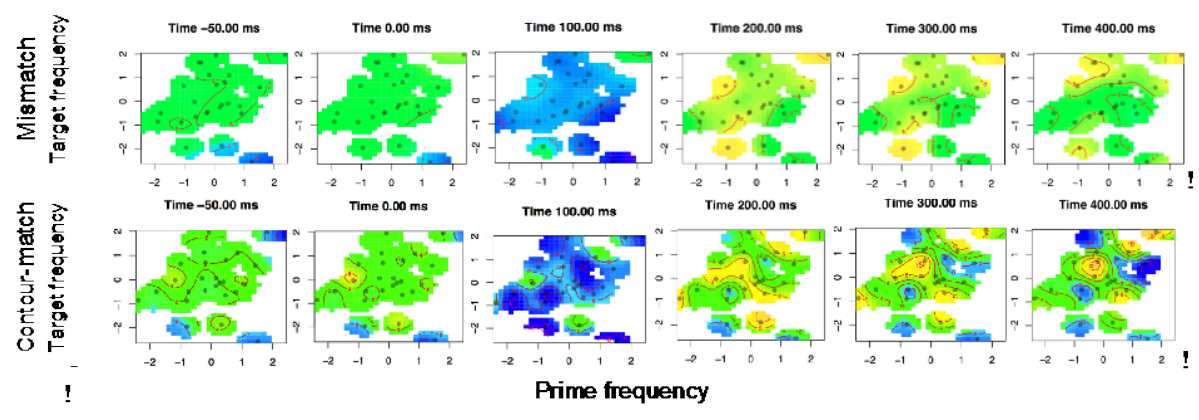

Figure 1. Topographical map of ERP amplitude at Fz.the interaction between prime frequency and target frequency over a series of time points $(-50 \mathrm{~ms}, 0 \mathrm{~ms}$, $100 \mathrm{~ms}, 200 \mathrm{~ms}, 300 \mathrm{~ms}, 400 \mathrm{~ms}$ ) for trials with the mismatch prime (top row) and contour match prime (bottom row) for electrode Fz. Each panel shows the interaction between Johnson transformed prime frequency (horizontal axis) and Johnson transformed target frequency (vertical axis). Yellow indicates relatively more positive amplitude; blue indicates more negative amplitude; green is at the intercept. The grey dots indicate the individual items. 


\section{Discussion}

The present results provide evidence for automatic retrieval of sub-phonemic information in visually processed masked prime words. The context provided by the tone of the second character of the prime determined whether the prime was rising, and therefore matched the target, or low, which mismatched the target. Model comparisons, model summaries and plots all showedsignificant differences in the pattern of ERPs when prime and target matched in tonal contour, compared to when they did not match. This demonstrates that even for very briefly presented masked primes, the context-specific phonetic form is processed. Moreover, activation of this context-specific variant influences processing of targets from a different tone category, demonstrating crosscategory phonological effects.

The analysis shows a complex interaction between prime type, prime frequency and target frequency. In the contour-match condition, the item characteristics came into play. When both prime and target frequency were high, there was increased negativity, suggesting an increased processing cost of resolving competition between activated candidates. In the mismatch condition, in contrast, there was little effect of the item frequencies. This last finding raises a methodological issue. In priming studies, interactions between prime and target frequency can have significant effects on ERP amplitude. Therefore, in ERP studies of language, it may be important to include item characteristics in the statistical analysis.

\section{Acknowledgements}

This work was supported by the European Research Council under the ERC Starting [Grant 206198] to YC.

\section{References}

Nixon, J., Chen, Y. and Schiller, N. (2015). Multi-level processing of phonetic variants in speechproduction and visual word processing: evidence fromMandarin lexical tones. Language, Cognition \& Neuroscience. 30 (5)491-505. DOI: $10.1080 / 23273798.2014 .942326$

Wood, S. (2006). Generalized additive models: an introduction with R. CRC press. 